\title{
KOOPERATIVER BERICHT VOM 7. DEUTSCHEN BIBLIOTHEKSIKONGRESS: „BIBLIOTHEKEN VERÄNDERN“ (LEIPZIG, 18.-21. MÄRZ 2019)
}

\author{
von Sebastian Aigner, Magdalena Andrae, Bruno Bauer, Susanne Blumesberger, \\ Olivia Kaiser und Markus Stumpf
}

Zusammenfassung: Der 7. Deutsche Bibliothekskongress hat von 18. bis 21. März 2019 in Leipzig stattgefunden. Das Motto der Konferenz, an der über 4.000 Personen (darunter 95 aus Österreich) teilgenommen haben, lautete „Bibliotheken verändern". Im vorliegenden kooperativen Bericht werden folgende Themen behandelt: Förderprogramm Fachinformationsdienste für die Wissenschaft, Langzeitarchivierung, Repositorien, Forschungsdaten und Forschungsdatenmanagement, Open Access, NS-Provenienzforschung, Qualitätsmanagement und Barrierefreiheit.

Schlagwörter: 7. Deutscher Bibliothekskongress; 108. Deutscher Bibliothekartag; Leipzig 2019; kooperativer Bericht

\section{COOPERATIVE REPORT OF THE 7TH GERMAN LIBRARIANS' CONGRESS: „LIBRARIES FOR CHANGE“ (LEIPZIG, MARCH 18-21, 2019)}

Abstract: The 7th German Librarians' Congress took place from 18 to 21 March 2019 in Leipzig. The motto of the conference, which was attended by more than 4.000 people (including 95 from Austria), was "Libraries for change“. This cooperative report covers the following topics: Specialised Information Services programme, long-term archiving, repositories, research data and research data management, open access, NS provenance research, quality management and accessibility.

Keywords: $7^{\text {th }}$ German Librarians' Congress; $108^{\text {th }}$ German Librarians' Day; Leipzig 2019; cooperative report

DOI: https://doi.org/10.31263/voebm.v72i1.2286

(c) Sebastian Aigner, Magdalena Andrae, Bruno Bauer, Susanne Blumesberger, Olivia Kaiser und Markus Stumpf

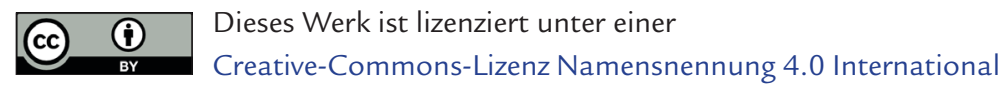




\section{Inhalt}

- Bibliotheken verändern: 7. Deutscher Bibliothekskongress in Leipzig (Bruno Bauer)

- Projektmanagement (Susanne Blumesberger)

- Fachinformationsdienste für die Wissenschaft - Evaluierung und Weiterentwicklung des DFG-Förderprogramms (Bruno Bauer)

- GND: Arbeit mit Normdaten (Sebastian Aigner)

- Von Blockchain bis Partizipation (Olivia Kaiser)

- Langzeitarchivierung und Repositorien (Susanne Blumesberger)

- Schwerpunkt Forschungsdaten und Forschungsdatenmanagement (Susanne Blumesberger)

- NS-Provenienzforschung (Olivia Kaiser und Markus Stumpf)

- Hands-on Lab: Neue Geschäftsmodelle und Workflows im Open Access (Magdalena Andrae)

- Hands-on Lab digital: Einsatz von Linux-Tools unter Windows für BibliothekarInnen (Magdalena Andrae)

- Hands-on Lab digital: DINI Metadata Crunch (Magdalena Andrae)

- Qualitätsmanagement-Forum 2019 (Bruno Bauer)

- Barrierefreiheit in (digitalen) Bibliotheken (Susanne Blumesberger)

- Podiumsdiskussion „Personal \& Kompetenzen - Wie gut sind wir?" (Susanne Blumesberger)

- Podiumsdiskussion „Herausforderungen bewältigt?" (Susanne Blumesberger)

\section{Bibliotheken verändern: 7. Deutscher Bibliothekskongress in Leipzig}

Der 7. Deutsche Bibliothekskongress - und zugleich der 108. Deutsche Bibliothekartag - fand unter dem Motto „Bibliotheken verändern“ von 18. bis 21. März 2019 im Congress Center Leipzig statt. Der Einladung von Bibliothek und Information Deutschland (BID) e.V., dem Dachverband der deutschen Bibliotheksverbände, zum Bibliothekskongress nach Leipzig, wo im Dreijahresrhythmus der größte nationale Fachkongress in Europa stattfindet, folgten 2019 ca. 4.000 Besucherinnen und Besucher, darunter mehr als 300 internationale Gäste. Bemerkenswert groß war auch die österreichische Abordnung, die laut Online-Teilnehmerverzeichnis 95 Personen zählte und sich wie folgt verteilte: 6 aus Bregenz, 17 aus Graz, 5 aus Innsbruck, 1 aus Klosterneuburg, 3 aus Linz, 9 aus Salzburg, 1 Sankt Pölten und 53 aus Wien.

Die Eröffnung des Kongresses fand am 18. März 2019 statt. Die Festrede wurde von Petra Laurentien Brinkhorst, Prinzessin der Niederlande, gehalten, die 2009 zur UNESCO Special Envoy on Literacy for Development ernannt 
worden ist. Vor rund 1.000 Teilnehmerinnen und Teilnehmern der Eröffnungsveranstaltung sprach sie über Rolle und Bedeutung von Bibliotheken. Die Niederlande waren auch das Gastland des 7. Bibliothekskongresses.

Unter dem Motto „Bibliotheken verändern“ widmete sich der Bibliothekskongress der Auseinandersetzung mit aktuellen Herausforderungen und wichtigen Zukunftsfragen des Bibliotheks- und Informationssektors; das Programm mit mehr als 260 Vorträgen gliederte sich in sechs Themenkreise:
(1) Politisch sein
(2) Strategisch handeln
(3) Content kuratieren
(4) Vielfalt bedienen
(5) Wissen lernen
(6) Zugänge ermöglichen

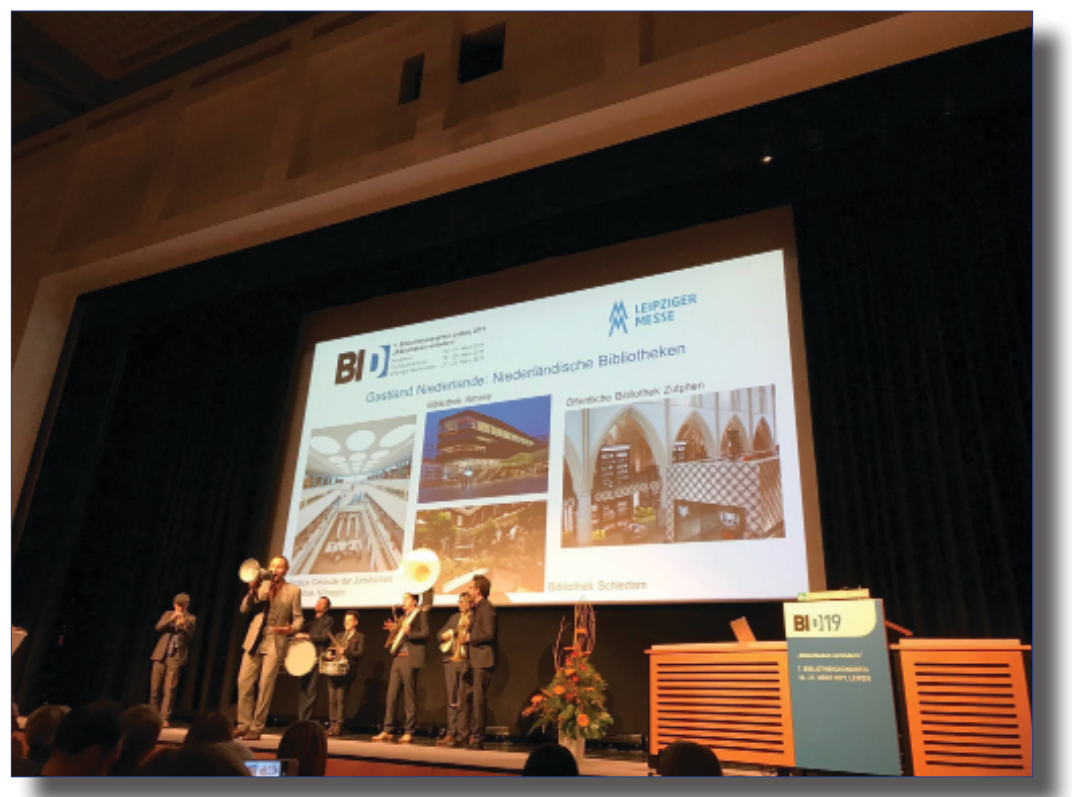

Abb. 1: Congress Center Leipzig: Cakewalkin'Babies bei der Eröffnung des 7. Deutschen Bibliothekskongresses (Foto: Susanne Blumesberger 2019)

Wie in der Vergangenheit bildete die begleitende Fachmesse, in der rund 150 internationale Firmen und Organisationen ihre neuesten Angebote und Services vorstellten und über aktuelle Trends informierten, eine wichtige Ergänzung zu den Vorträgen und zum wissenschaftlichen Programm. Ein abschließendes Highlight stellte für viele Tagungsteilnehmerinnen und 
-teilnehmer, wie in den vergangenen Jahren, die Möglichkeit dar, am Donnerstag kostenfrei die Buchmesse zu besuchen, die im Anschluss an den Bibliothekskongress von 21. bis 24. März 2019 stattfand und an der sich 2.535 Aussteller aus 46 Ländern beteiligten, zu besuchen. Mit 286.000 Besucherinnen und Besuchern konnte die Buchmesse 2019 einen neuen Besucherrekord aufstellen.

Aufgrund der Fülle der Vorträge und der zahlreichen parallelen Vortragsblöcke am Bibliothekskongress wäre das Verfassen eines repräsentativen Tagungsberichtes für eine Einzelperson nicht möglich. Aus diesem Grund ist es in den letzten Jahren schon zur Tradition geworden, dass Tagungsteilnehmerinnen und -teilnehmer die Aufgabe übernehmen, aus einer subjektiven Perspektive ihre Eindrücke von einzelnen besuchten, besonders informativen Sessions in kurzen Reportagen zusammenzufassen. Diese Berichte bilden die Grundlage für den vorliegenden kooperativen Tagungsbericht und vermitteln in ihrer Gesamtheit einen guten Eindruck vom vielfältigen Programm des 7. Bibliothekskongresses in Leipzig. Viele der gezeigten Präsentationen und in der Folge vorgestellten Vorträge stehen online am BIB-Opus-Server zur Verfügung (https://opus4.kobv.de/opus4-bib-info/ solrsearch/index/search/searchtype/collection/id/16963).

Koordination des Berichts: Bruno Bauer

\section{Projektmanagement}

In der Session PROJEKTMANAGEMENT AKTUELL berichtete zunächst Gabriella Padovan (Zürich) über die „Erneuerung der ProjektmanagementMethode an der ETH-Bibliothek". Nach einer testweisen Anwendung des agilen Rahmenwerks "Scrum" entschied die Geschäftsleitung der ETHBibliothek, die klassische Projektmanagement-Methode weiterzuentwickeln. Padovan berichtete in ihrem Vortrag von den Erfahrungen bei der Einführung von agilen Methoden, was zu mehr Transparenz, Visualisierung, Zugänglichkeit und Zusammenarbeit im Netzwerk geführt hat. Die zahlreichen Reflexionen tragen dazu bei, Verbesserungsmöglichkeiten zu sehen.

„Das TIB AV-Portal setzt auf das agile Management-Framework Scrum" - unter diesem Titel informierte Sven Strobel (Hannover), dass 2016 auch die Technische Informationsbibliothek (TIB) Scrum im Bereich IT-Entwicklung eingeführt wurde. Im August 2018 wurde das Videoportal (TIB AV-Portal) ebenfalls auf Scrum umgestellt. Eine zu konkrete Planung ist dabei eher hinderlich, allerdings sind kurze intensive Treffen sehr hilfreich. 
Im Anschluss daran informierten Sabine Schrimpf und Sandra Blanck (Frankfurt) über die Erfahrungen, die im Rahmen von „10 Jahre Projektworkflow an der Deutschen Nationalbibliothek" gemacht wurde. Innovative Vorhaben, Anpassungen an Geschäftsgänge und andere einmalige, nicht wiederkehrende Vorhaben größeren Umfangs nach einem intern festgelegten, an Standards orientierten Verfahren werden als Projekte organisiert, wobei die oberste Leitungsebene der DNB als „Projektboard“ fungiert.

Susanne Blumesberger

$* * * * * *$

\section{Fachinformationsdienste für die Wissenschaft - Evaluierung und Weiter- entwicklung des DFG-Förderprogramms}

Gudrun Oevel (Paderborn) und Joachim Kreische (Dortmund) präsentierten Ergebnisse der „Evaluierung des Förderprogramms Fachinformationsdienste für die Wissenschaft" sowie „Empfehlungen der Evaluierungskommission I: Ausrichtung des Programms und Umsetzung der Förderung" und „Empfehlungen der Evaluierungskommission II: Zusammenwirken der Fachinformationsdienste".

2012 wurde vom Hauptausschuss der Deutschen Forschungsgemeinschaft (DFG, https://www.dfg.de/) das Förderprogramm „Fachinformationsdienste für die Wissenschaft“(FID, https://www.dfg.de/foerderung/ programme/infrastruktur/lis/lis_foerderangebote/fachinfodienste_wissenschaft/index.html) verabschiedet, mit dem ein Prozess der Weiterentwicklung der im Jahr 1949 eingerichteten Förderung der „Sondersammelgebiete" an wissenschaftlichen Bibliotheken in Deutschland gestartet wurde. Während die Bibliotheken die Grundversorgung abdecken, kommt den FIDs die Aufgabe zu, eine qualifizierte Informationsversorgung in Ergänzung zur Grundversorgung zu leisten. Ziele der Förderung der FIDs durch die DFG sind (1) die Ermöglichung des überregionalen und schnellen Zugriffs zu Spezialliteratur und die Entwicklung weiterer fachspezifischer Informationsdienstleistungen, (2) die Adressierung spezifischer Interessen der Fach-Communities sowie (3) als besonderer Schwerpunkt der Zugang zu digitalen Medien.

Um zu überprüfen, inwieweit diese Vorgaben erreicht wurden, wurde eine Evaluierung in Auftrag gegeben. Deren Zielsetzung war es, eine Programmbewertung durchzuführen, Empfehlungen für die weitere Entwicklung abzugeben sowie eine Diskussionsgrundlage für Lösungsansätze hinsichtlich einer nachhaltigen Finanzierung zu schaffen. Im Rahmen ei- 
ner Online-Befragung aller 35 Fachinformationsdienste erfolgte eine Bestandsaufnahme, deren Ergebnisse in acht Fallstudien vertieft wurden. Die Evaluationsstudie wurde unter Einbeziehung eines externen Dienstleisters im August 2018 vorgelegt, die Empfehlungen der Kommission zur Weiterentwicklung des Programms wurden als Stellungnahme im Oktober 2018 fertig gestellt. Studie und Empfehlungen bilden die Grundlage für die zukünftige Programmgestaltung, die vom Ausschuss für Wissenschaftliche Bibliotheken und Informationssysteme (AWBI, https://www.dfg.de/dfg profil/gremien/hauptausschuss/wissenschaftliche_bibliotheken/index. html), einem Beratungsgremium der DFG, betrieben wird.

In die Evaluation einbezogen wurde die Perspektive der FIDs, der (potenziellen) Nutzerinnen und Nutzer sowie der (Nicht-FID)Bibliotheken. Bemerkenswerte Ergebnisse waren unter anderem die große Rolle, die Interdisziplinarität an der Hälfte der FIDs spielt, sowie die starke Zunahme des Stellenwertes von Open Access-Fachrepositorien, von publikationsunterstützender Beratung bei Erst- und Zweitveröffentlichung, von Rechteerklärung, von Open Access sowie von Hostings und Erschließung von Zeitschriften. Die bisherige Zusammenarbeit wurde sowohl von den FIDs als auch von den wissenschaftlichen Bibliotheken (ohne FID) überwiegend positiv bewertet.

In ihrer Stellungnahme zur Evaluierung der Förderung der einzelnen FIDs wurde von der Kommission festgehalten, dass die Richtung stimmt und die Chance zur Strukturbildung und zur Etablierung von Standards besteht. Auch wenn fachspezifische Lösungen angestrebt werden, so erweisen sich die Strukturen dennoch als effizient. Empfohlen wurde die Optimierung der Zugänglichkeit durch nutzernahe Nachweissysteme, die Nutzung von Multiplikatoren, ein maximaler Verzicht auf Zugangsrestriktionen sowie ein transparenter und einfacher Zugang zu den Angeboten. Im Bereich der E-Medien wurde das Konzept des „E-preferred“ begonnen, welches aber derzeit am Markt schwer durchzusetzen ist. Anstelle von DRM sollte Open Access forciert werden. Wichtig ist es auch, die Nachhaltigkeit der E-Ressourcen zu sichern. Dies soll durch Lizenzierung von Archivrechten, Sicherstellung der Langzeitverfügbarkeit (durch Einbindung in nationales Hosting, aber auch Portico und CLOCKSS) sowie Etablierung von hochwertigen, standardisierten, interoperablen und dauerhaft verfügbaren Metadaten gewährleistet werden.

Aus Sicht der Kommission ergeben sich für die FIDs folgende Herausforderungen: (1) Festlegung der zukünftigen Ausrichtung des Programms der Umsetzung der Förderung, (2) langfristige Finanzierungsperspektive für Kernaufgaben eines FID und (3) Zusammenwirken der FIDs untereinander. 
Für 2019 sind noch Arbeiten an der Weiterentwicklung des FID-Programms geplant, dessen finale Version im Februar 2020 vom AWBI vorgelegt werden soll. Im Juni 2020 soll das weiterentwickelte FID-Programm vom Hauptausschuss der DFG verabschiedet werden, um so den Bestand der FIDs als wichtige Säule der Fachinformation in Deutschland abzusichern.

Bruno Bauer

$* * * * * *$

\section{GND: Arbeit mit Normdaten}

Im Gegensatz zu den vergangenen Jahren, in denen der GND-Block jeweils parallel zu anderen, das gleiche Publikum ansprechenden, Veranstaltungen (vornehmlich im Themenfeld RDA) programmiert war, erfreute sich die Veranstaltung diesmal eines regen Zulaufes. Zahlreiche Personen ergatterten nicht einmal mehr einen Stehplatz, es hätte wohl gut und gerne ein doppelt so großer Saal gefüllt werden können, was wohl als Zeichen für die steigende Relevanz der GND gewertet werden kann. Insgesamt umfasste der dreistündige, von Silke Schomburg, vom Hochschulbibliothekszentrum des Landes Nordrhein-Westfalen in Köln moderierte, Veranstaltungsblock sechs Vorträge.

Den Anfang machte Jürgen Kett mit „GND-Erschließung der Zukunft: Wechselwirkungen mit dem RDA-Kosmos, den nicht-bibliothekarischen Welten und dem Wikiversum". Als Leiter der Arbeitsstelle für Standardisierung an der Deutschen Nationalbibliothek in Frankfurt am Main ist er prädestiniert dafür, einen Ausblick auf die bevorstehenden Herausforderungen in Zusammenhang mit der Öffnung der GND für außerbibliothekarische Nutzer_innenund Mitarbeiter_innengruppen, aber auch die generelle Modernisierung der GND in Bezug auf Datenmodell, Datenformat etc. zu geben. Obwohl hier unterschiedliche Anforderungsprofile und Konzepte aufeinandertreffen und manche der Standards sich noch in Fluss befinden - bspw. IFLALRM und seine Auswirkungen auf RDA - zeichnete Kett ein optimistisches Bild der bevorstehenden Entwicklungen. Erste positive Erfahrungen und Zwischenergebnisse, die diesbezügliche Projekte in den letzten beiden Jahren erbracht haben, bringen ihn zur Überzeugung, dass sich die Probleme, unter anderem durch eine Modularisierung der GND (Core/Plus Datenmodell), lösen lassen werden und die beteiligten Communities allesamt von einer gemeinsam genutzten und gepflegten GND profitieren werden. 
Zu bewerkstelligen wird dies jedoch nicht ohne eine gewisse Flexibilität, auch seitens der Bibliothekswelt, sein.

Eines der oben erwähnten Projekte ist das von der DFG geförderte GND4C - GND für Kultureinrichtungen, mit welchem sich die drei folgenden Vorträge beschäftigten. In „\#Neuland - Museen und Archive als gleichberechtigte Partner in der GND-Kooperative. Die zentralen Herausforderungen des Projekts GND4C" gab erneut Jürgen Kett, diesmal Barbara Fischer von der Deutschen Nationalbibliothek in Frankfurt am Main vertretend, zunächst einen Überblick über die Aktionsfelder und Projektziele. Die Pflege der GND durch die beteiligten bibliothekarischen Einrichtungen beruht auf etablierten Organisationsstrukturen, abgestimmten Regeln und Workflows sowie speziellen technischen Umgebungen. Eine künftige Zusammenarbeit in punkto GND zwischen Bibliothekswesen einerseits und Archiv- und Museumswesen, Mediatheken und wissenschaftlichen Einrichtungen andererseits bedarf beidseitiger Anpassungen in all diesen Bereichen. Um dies zu bewerkstelligen wurden folgende vier Ziele definiert: der nachhaltige Aufbau einer sparten- und fächerübergreifenden Organisation, die Weiterentwicklung des Datenmodells und der Regeln im Hinblick auf nicht-bibliothekarische Anwendungskontexte, die Bereitstellung von Schnittstellen und Werkzeugen zur Unterstützung nicht-bibliothekarischer Anwendungskontexte und die Stärkung der Kommunikation mit den verschiedenen Interessengruppen über verschiedene Kommunikationskanäle sowie die Sichtbarmachung des GND-Netzwerks. Im zweiten Teil des Vortrages wurden die bisherigen Fortschritte und geplanten Entwicklungen in Bezug auf den Aufbau bzw. die Anpassung der Organisationsstruktur der beteiligten Communities, die Adaption des GNDDatenmodells an die Erfordernisse eines breiteren Nutzer_innenkreises und die Entwicklung von Werkzeugen zur möglichst effizienten Datenpflege, mit speziellem Fokus auf dem Abgleich bestehender Normdatenbestände aus Museen, Archiven etc. gegen die GND, präsentiert.

Konkret werden jeweils unterschiedliche Aspekte der Projektziele anhand von Fallbeispielen behandelt und praxistaugliche Ergebnisse erarbeitet. Den Anfang machten Susanne Laux vom Landesarchiv BadenWürttemberg in Stuttgart und Martha Rosenkötter vom Deutschen Dokumentationszentrum für Kunstgeschichte - Bildarchiv Foto Marburg mit dem Vortrag „Stadt, Land, Bauwerk. Geografika auf dem Prüfstand. Ein GND4CWerkstattbericht". Sie zeigten, wie im wichtigen Bereich der Geografika - neben den zu erschließenden Ressourcen haben auch diverse anderen GND Entitäten selbst geografische Bezüge, welche sich in Form interner GNDVerlinkungen äußern - versucht wird, die GND als übergreifende Referenzdatei zu etablieren. Schwerpunkte sind hierbei in Bezug auf die „herkömm- 
lichen" Geografika der quantitative Ausbau des Datenbestandes, inklusive Erhöhung der Granularität und Georeferenzierung, sowie das Eingehen auf besondere Bedürfnisse von Archiven, Museen etc. in punkto der historischen Dimension von Geografika. Was die in der GND ebenfalls zur Entität Geografikum gehörenden Bauwerke und Monumente bzw. sogenannten ortsfesten Kunstwerke betrifft, geht es vornehmlich darum, die in Bibliotheken sowie Museen, Archiven etc. vorherrschenden Konzepte in Bezug auf diese in Einklang zu bringen. Der Fokus der Entwicklungsarbeit in diesen beiden Fallbeispielen liegt demnach einerseits auf der Weiterentwicklung des GND-Datenmodells, aber auch des Regelwerkes, um die, durch die neu hinzukommenden Communities nun relevanten, Dimensionen der Geografika und Bauwerken etc. abbilden zu können, und andererseits auf der Erarbeitung von Workflows und Konzepten zum Mapping bestehender Datenbestände auf die GND.

Den Fallbeispielen „Registrierung objektbeschreibender Sachbegriffe der Museumsdokumentation“ und „Verzeichnung von Künstlerinnen und Künstlern für die museale Forschung" widmeten sich Jens Lill vom Bibliotheksservice Zentrum Baden-Württemberg in Konstanz und Jutta Lindenthal vom digiCULT-Verbund eG in Kiel in ihrem Beitrag „Wer, wie, was? Personeninformationen und Sachbegriffe aus Museen in der GND und ihre Repräsentation im GND-Datenmodell. Ein GND4C-Werkstattbericht". Obwohl die beiden Entitätentypen sowohl in der Bibliotheks- als auch in der Museumswelt für die Erschließung der Bestände von großer Bedeutung sind, finden in letzterer vornehmlich andere Vokabulare (Internationales wie Getty-ULAN und Getty-AAT aber auch selbstgestrickte Normdatenbanken) Anwendung. Da auf Seiten der Museen durchaus reges Interesse an der Vernetzung der Bestände mit denen anderer Kultureinrichtungen besteht (Stichwort DDB oder Europeana), widmen sich die beiden Fallbeispiele den Anpassungen, die GND-seitig nötig wären, um Museen zu ermöglichen die GND zu nutzen bzw. sich an deren Pflege zu beteiligen, sei es nun direkt oder indirekt in Form von Konkordanzen. Größter Hemmschuh ist, wie schon bei den Geografika, auch in Bezug auf Personen und Sachbegriffe, die mangelnde Abdeckung des benötigten Vokabulars durch die GND. Dies erklärt sich daraus, dass die GND nie den Anspruch hatte ein vollständiger Thesaurus zu sein oder auch nur einzelne Begriffsfelder, geografische Räume etc. vollständig abzudecken. Sie war bis dato lediglich eine Datenbank, in die, je nach Bedarf an einzelnen Bibliotheken, Datensätze eingebracht wurden, welche dann von den Partnerinstitutionen nachgenutzt werden konnten. Um dies zu ändern sind neben Änderungen am Datenmodell, um die Bedürfnisse der Museen abdecken zu können, auch organisatorische bzw. re- 
daktionelle Absprachen zwischen den Communities sowie leistungsfähige Schnittstellen zwischen den Systemen von Nöten. Im Bereich Personen kann die GND hiervon einerseits durch einen Ausbau des Datenbestandes in Form der Einspielung von noch nicht vorhandenen Personen-Datensätzen aus den Museums-Datenbanken profitieren, und auch die Qualität der vorhandenen GND-Datensätze kann gehoben werden, indem der Altbestand mit Zusatzelementen aus dem Museumsbereich angereichert wird und im selben Zuge auch Dubletten getilgt werden. Andererseits kann die Analyse des GND-Datenbestandes, -Datenmodells und -Regelwerks im Zuge des Projektes Inkonsistenzen ans Tageslicht fördern, die auf konzeptioneller Ebene bereinigt werden können. Bei den Sachbegriffen besteht, neben dem augenscheinlichen quantitativen Gewinn durch die Einspielung neuer Begriffen aus der Museumswelt, auch beim bestehenden Vokabular die Möglichkeit dies qualitativ um die zahlreichen, in den Museums-Vokabularen vorhandenen, Relationen anzureichern, was den Thesaurus-Charakter dieses GND-Teilbestandes stärken würde.

Angela Vorndran und Stefan Grund, beide von der Deutsche Nationalbibliothek in Frankfurt am Main, widmen sich in ihrem Vortrag dem Thema der maschinellen Erschließung von Ressourcen mittels Normdaten, genauer gesagt, der Evaluierung dieser automatisierten Erschließungsprozesse. In „Die Maschine überwacht die Maschine: Wie maschinelle Verfahren automatisierte Prozesse verbessern können" erläutern sie, dass eine intellektuelle Kontrolle der Ergebnisse maschineller Erschließung aufgrund der enormen Menge erschlossener Ressourcen nur unvollständig erfolgen kann. Deshalb wurden Verfahren zur ebenfalls maschinellen Generierung von Kennzahlen entwickelt, welche einerseits der Vorhersage der zu erwartenden Qualität der automatisierten Prozesse in Bezug auf eine bestimmten Titeldatensatz und andererseits der Beurteilung des Erschließungsergebnisses im Nachhinein dienen. Auf Basis dieser Kennzahlen, die für die Sachgruppenvergabe und die Bildung von Werkclustern vergeben werden, sollen sowohl die maschinellen Verfahren gezielter eingesetzt, als auch die intellektuelle Ex-post-Kontrolle auf jene Datensätze konzentriert werden, bei denen die signifikanteste Verbesserung des Ergebnisses zu erwarten ist.

Im letzten Referat des GND-Blocks „Erschließung für den deutschsprachigen Raum: Fazit und Ausblick der DNB" zog Elisabeth Niggemann, Generaldirektorin der Deutschen Nationalbibliothek, Bilanz über die Entwicklung des Erschließungsbereiches in den vergangenen Jahren und gab einen Ausblick auf die, ihrer Ansicht nach, wichtigsten Herausforderungen der nächsten Jahre. Laut Niggemann muss sich die Erschließung von Ressourcen ständig weiterentwickeln, um sich an die, sich laufend verändernden, 
Anforderungen der Informationswelt anzupassen. Auf Regelwerks-, Format und organisatorischer Ebene ist dies in der Vergangenheit mittels der Einführung von RDA, MARC21 als Austauschformat und der GND als gemeinsamer Normdatenbank für Formal- und Inhaltserschließung einerseits und gemeinschaftlich geführter Datenbank für den deutschsprachigen Raum andererseits gut gelungen. Für die Zukunft sieht sie die, vom Standardisierungsausschuss koordinierte, Mitarbeit an der Entwicklung internationaler Standards sowie die verstärkte Zusammenarbeit der Institutionen aus Deutschland, Österreich und der Schweiz in punkto intellektuelle Erschließung und die Hebung des großen Potentials der automatisierten Erschließungsverfahren als wichtigste Arbeitsbereiche, um der von Bibliotheken geleisteten Arbeit weiterhin gesellschaftliche Relevanz zu sichern.

\section{Sebastian Aigner}

$* * * * * *$

\section{Von Blockchain bis Partizipation}

Ralph Stockmann (Berlin) stellt in seinem Vortrag „Wie man vom Intranet aus die Welt verbessern kann. Bibliotheken als Plattform gesellschaftlicher Teilhabe" die These auf, dass der physische Bestand von Bibliotheken durch die fortschreitende digitale Transformation in den nächsten zehn bis fünfzehn Jahren an Bedeutung verlieren werde. Eingeleitet mit der Polemik „too old to fail“ stellt sich der Referatsleiter der Abteilung Innovations-Managements der Frage, wie Bibliotheken mit dieser Herausforderung umgehen können.

War noch 2006 das kanonische Wissen im Brockhaus verortet, so wurde dieser 2010 bereits von Wikipedia abgelöst - Stockmann ist für seine pointierte Frage bekannt, warum denn Bibliothekarlnnen nicht Wikipedia erfunden hätten. Bereits für 2019 konstatiert Stockmann YouTube als das Nachschlagewerk Nummer Eins und schließt daraus, dass „Information zunehmend vernetzt, dezentral und entlang von Kommunikationsprozessen entsteht".

Bibliotheken können sich in diesem Prozess einklinken und als „dritter Ort" den Weg der Digitalisierung beschreiten. Zugang zu freier Infrastruktur sichert Teilhabe und Inklusion. Stockmann plädiert für den „dritten Ort" Bibliothek das Setzen eines Gegengewichts zu kommerziellen (digitalen) Produkten und schlägt hierfür die strategische Partnerschaft mit Open Source Entwicklungen vor. Die politische Dimension verdeutlicht er 
durch Hinweise auf Datenskandale der letzten Jahre und die kritisch zu betrachtenden Entwicklungen, wer wo welche Daten speichert.

Bibliotheken können eigene Entwicklungen in wenigen Fällen selbst leisten, haben doch Open Source Projekte einiges zu bieten: von Tests, Bugreports, Dokumentation bis Hosting und Betrieb, Vermittlung, Schulung und dem Sammeln von User Experience. Als weiteren Pluspunkt bringt Stockmann die „digitale Allmende“ ins Rennen: Bibliotheken haben keine Hidden Agenda, erschließen neue Nutzergruppen und verfügen über die spezifische „Aura der Bibliothek“.

Wie soll nun das Intranet die Welt verbessern können? Nach Stockmann eignet sich das an vielen Institutionen vorhandene Tool als Spielwiese und Qualifizierungsmaßnahme für Bibliotheksangestellte. Als „Stufenmodell von Öffentlichkeit" kann ein Intranet herangezogen werden, um neue Open Source Tools einzuführen, in Arbeitsgruppen und später in der Institution und anschließend einem Institutionenverbund zu testen. Nach dem Sammeln von Erfahrungen, können erste Nutzergruppen „überzeugt werden", um in einem weiteren Schritt eine breite (weltweite) Öffentlichkeit zu erreichen.

In seinem Vortrag listet Stockmann für Anwendungen von Fileservern, Terminfindungstools bis Online Offices zu bekannten Plattformen Alternativen auf und präsentierte auch gleich den digitalen bibliothekarischen Raum https://openbiblio.social, der ähnlich wie der Kurznachrichtendienst Twitter funktioniert. Dahinter steckt die Open Source-Software Mastodon, die als Alternative zu Twitter auf dezentralen Servern läuft. Zielgruppe sind deutschsprachige KollegInnen aus wissenschaftlichen und öffentlichen Bibliotheks- und Informationseinrichtungen. Die SBB hostet den Raum ohne Branding, der Teil der openbiblio-Familie https://openbiblio.eu ist, wo etwa auch die bibliothekarische Jobplattform https://jobs. openbiblio.eu/ hinzuzählt.

Alle Interessierten wurden im Vortrag herzlich eingeladen unter https:// openbiblio.social nach erfolgter Registrierung über ihre Themengebiete zu „tröten“, der Aufruf sei hier nun an die LeserInnen weitergeleitet!

Jens Bemme und Martin Munke (Dresden) stellten in ihrem Vortrag die Frage „Macht Citizien Science glücklich? Bürgerwissenschaften in wissenschaftlichen Bibliotheken“. Nach den Vortragenden sind Sätze wie „Citizien Scientists nerven“ oder „Citizen Scientists halten uns von echter Wissenschaft ab"gängige Vorbehalte von Wissenschaftlerlnnen gegenüber der bürgerbeteiligten Forschung. Bemme und Munke machen sich dafür stark, dass es aber ohne Citizen Scientists nicht ginge und verweisen auf die lange Tradition von bürgerbeteiligter Forschung seit dem 19. Jahrhundert, in dem 
Bibliotheken stets ein Teil dieses Prozesses waren. Zur Untermauerung dient das Beispiel der Heimatforschung und der SLUB-gehosteten Plattform www.saxorum.de, die insbesondere von Linked Open Data, wie der Georeferenzierung im Virtuellen Kartenforum 2.0., profitiert.

Citizien Science macht glücklich (Dr. Matthias Nuss, Senckenberg Gesellschaft für Naturforschung Dresden), wenn es für die „echten“ Wissenschafterlnnen keine Nebenbeschäftigung ist und Themen und Projekte von ihnen selbst angestoßen werden. Beidseitiges Lernen erfolgt durch die Schaffung von Verbindungen und bei der Zusammenarbeit von Wissenschafterlnnen und Bürgerlnnen. Die SLUB Dresden nimmt sich dem Thema Citizien Science im Zuge ihrer Strategieentwicklung bis 2025 an.

Maximiliane Okonnek (Zürich) nahm sich in ihrem Vortrag „(Scientific) Bibs on the Blockchain - Abgesang mit Zukunftsperspektive" vor, die Technologie Blockchain dem Publikum näherzubringen und fragte nach der Bedeutung für Bibliotheken.

Relativ simpel klingt die Erklärung, dass ein „Block“ eine digitale Information und eine „Chain“ eine Datenbank sei. In einem Peer-to-Peer Netzwerk werden die Informationen organisiert, Ketten von Blöcken gebildet, kryptografische Verfahren sowie ein Konsens- und Anreizsystem angewandt - möglicherweise benötigt man hier bereits etwas mehr Vorstellungskraft.

Okonnek listet einige Gründe auf, warum Bibliotheken sich mit Blockchain auseinandersetzen sollten. Naheliegend klingt die Begründung, dass Blockchain eine Informationsinfrastruktur darstellt und somit per se für Bibliotheken als Informationseinrichtungen - insbesondere im Hinblick auf Publikationsstrukturen - interessant ist. Im Weiteren ist die Vortragende davon überzeugt, dass Kryptowährungen ein Thema für Bibliotheken werden.

Als Beispiele für den Einsatz von Blockchain in den Wissenschaftscommunities lassen sich Themen wie Reproduzierbarkeit (unverändertes Dokumentieren), Kollaboration (Management von Zugriffsrechten, Log Files, etc.), Publizieren (Logging von Publikationsereignissen und Beiträgen wie Beobachtungen, Null-Ergebnisse, Preprints etc.) sowie Forschungsförderung (alternative Fördermechanismen) aufzählen.

Dennoch bestehen viele Hindernisse, die Okonnek nicht ungenannt lässt. Zunächst besteht eine hohe Komplexität in technischer und konzeptioneller Weise. Neben dem hohen Stromverbrauch fehlen Standards und User Experience, darüber hinaus bleiben rechtliche Aspekte (noch) ungeklärt. Zu dem verhindert die oftmals fehlende Kompatibilität der wissenschaftlichen Informationsinfrastrukturen den Einsatz von BlockchainTechnologien. 
Abschließend plädiert der Managing Director des ETH Library Lab für einen hybriden Ansatz, in dem die geeignetsten der verfügbaren Technologien miteinander verschmolzen werden.

Olivia Kaiser

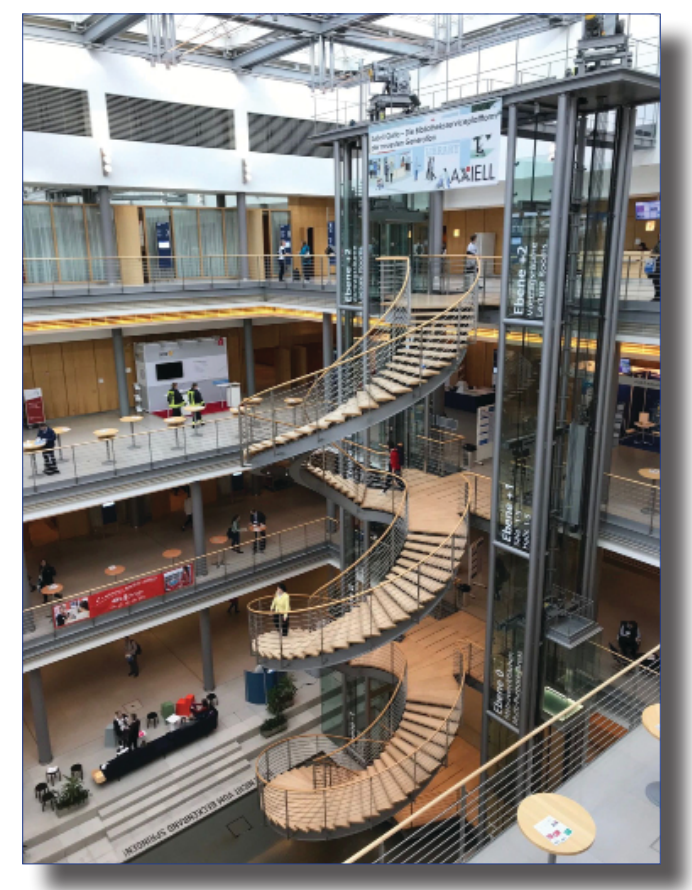

Abb. 2: Congress Center Leipzig: Treppenhaus (Foto: Susanne Blumesberger 2019)

\section{Langzeitarchivierung und Repositorien}

Hildegard Schäffler (München) beschäftigte sich in ihrem Beitrag mit dem Thema „Nationales Hosting elektronischer Ressourcen: Von der Konzeptentwicklung zur Implementierung". Ziel des von der DFG geförderten Projekts war die Entwicklung eines bundesweit abgestimmten Konzepts zur Sicherung des dauerhaften, von Verlagsplattformen unabhängigen Zugriffs auf lizenzierte Ressourcen. Das Projekt NatHosting baut auf der an der Universität Stanford entwickelten Software LOCKSS auf, das von voneinander unabhängigen vier Sicherungen der Inhalte ausgeht. Das Fortsetzungsprojekt NatHosting II (2018-2021) hat die Implementierung dieses Konzepts zum Ziel. Projektpartner sind die Bayerische Staatsbibliothek, FIZ Karlsru- 
he - Leibniz-Institut für Informationsinfrastruktur, die Universitätsbibliothek Johann Christian Senckenberg Frankfurt am Main, die Bibliothek des Karlsruher Instituts für Technologie (KIT), das Institut für Bibliotheks- und Informationswissenschaft der Humboldt-Universität zu Berlin sowie die Universitätsbibliothek Erlangen-Nürnberg.

Monika Zarnitz (Kiel) informierte über „CoreTrustSeal und nestor-Siegel: Lessons learned aus den Zertifizierungen für die digitale Langzeitarchivierung". Beim CTS sind Fragen technischer Art, aber auch institutioneller Art zu beantworten. Beim Nestor-Siegel sind 24 Fragen zur Sammlungsstrategie, Preservation Policy, Finanzierungsfragen, aber auch zum Personalentwicklungskonzept und zur Sicherheit zu beantworten. Insgesamt bedeutet eine Zertifizierung einen großen Aufwand, allerdings bringt sie natürlich auch einen Zusatznutzen, nämlich die Sichtbarkeit nach außen und eine Reflexion über die eigenen Dienste.

„Preservation-as-a-Service: Das Dienstleistungsangebot zur Langzeitarchivierung der TIB" lautete das Thema von Thomas Bähr (Hannover). Die TIB Hannover stellte das Dienstleistungsangebot zur Langzeitarchivierung (LZA) Preservation-as-a-Service vor. Die TIB stimmt alle Schritte mit den Kunden $a b$, berät sie in Fragen der Aufbereitung der Inhalte und deren Strukturierung, führt notwendige Prüfroutinen durch und archiviert die ihnen übergebenen Inhalte. Nach dem Ingest übernimmt die TIB das so genannte Preservation Management, was ein umfangreiches TechnologyWatch und Community-Watch bedeuten. Werden technologische Veränderungen im Umfeld festgestellt, die Auswirkungen auf die archivierten Daten haben, so informiert die TIB, die Teil des nationalen Hostings ist, ihre Kunden und gibt Empfehlungen zum Umgang mit den Dateien. Gemeinsam werden dann Erhaltungsmaßnahmen festgelegt, die dann entweder durch die TIB oder aber bei Bedarf auch außerhalb des Systems durch den Kunden durchgeführt werden. Das Angebot umfasst unter anderem das Bereitstellen von Soft- und Hardware, Qualitätskontrollen und Backup-Dienste. Die LZA ist bereits im Ausbildungsbereich verankert. Fazit ist, dass LZA Chefsache sein sollte, Verantwortlichkeiten sollten definiert sein, Policies sollten transparent sein, juristische Fragen geklärt werden. Die wichtigste Conclusio: LZA ist kein Zustand, sondern ein Prozess!

Im letzten Vortrag dieser Session wurde von Daniel Scharon (Konstanz) das Thema "Software im Forschungsprozess: Archivierung und Publikation von Softwareartefakten mit SARA“ besprochen. Der SARA-Server (https://saraservice.org) unterstützt Forschende bei der Archivierung und Publikation von Software und Softwareartefakte. Dabei werden die verschiedenen 
Versionen festgehalten und Persistent Identifier vergeben. Der Quellcode des SARA-Servers, das Bindeglied zwischen GitLab bzw. GitHub, Archiv und Repositorium, wird unter einer Open Source Lizenz veröffentlicht. Ziel dabei ist Softwareartefakte langfristig verfügbar zu machen, zum Beispiel kleine Hilfsskripte.

Susanne Blumesberger

\section{Schwerpunkt Forschungsdaten und Forschungsdatenmanagement}

Wie schon in den letzten Jahren lag der Schwerpunkt der Deutschen Bibliothekskongresse bzw. -tage auf dem Thema Forschungsdaten. Dazu wurden mehrere Sessions zum Themenkreis CONTENT KURATIEREN angeboten.

Eine von Klaus-Rainer Brintzinger (München) moderierte Session „FORSCHUNGSDATEN" beschäftigte sich mit Forschungsdatenmanagement an unterschiedlichen Universitäten.

Unter dem Titel „Forschungsdatenmanagement - ein lösbare Lernaufgabe?! Identifikation und Entwicklung relevanter Kompetenzen" wurde Katarina Blask (Trier) eine FDM-Kompetenzmatrix sowie das dazu passende FDM-Qualifizierungskonzept - vom durch das BMBF geförderten Projekts „Prozessorientierte Entwicklung von Managementinstrumenten für Forschungsdaten im Lebenszyklus" (PODMAN; www.fdm.uni-trier.de) - vorgestellt. Prozesse sollen über Rahmenbedingungen effizienter gestaltet werden. Relevant scheinen hier vor allem die Entscheidungstabellen zu sein, die zeigen was man schon hat und was die Institution noch nicht erfüllt. Wie immer spielen die (fehlenden) Ressourcen aber auch fehlende Kompetenzen und Qualifikationen eine große Rolle. Eine Kompetenzmatrix zeigt den Bedarf auf.

Anschließend beschäftigte sich Jens Dierkes (Köln) mit dem Thema „Interdisziplinäres, komplexes Forschungsdatenmanagement am Beispiel der Universität zи Köln". An der Universität Köln, die durch eine dezentrale Organisationskultur geprägt ist, stehen zentrale Einrichtungen wie Rechenzentrum, Bibliothek, aber auch das Dezernat Forschungsmanagement im Wechselspiel untereinander und mit einer Zahl von weiteren Akteuren auf dem Campus. Hier zeigte sich, dass zunächst die vielseitigen Bedarfe abwägend relativiert werden müssen und gleichzeitig eine am Bedarf ausgerichtete Angebotsentwicklung zu verfolgen ist. Es ist geplant, ein Kompetenzzentrum für Forschungsdaten zu etablieren. Dazu werden Dialoge auf unterschiedlichen Ebenen initiiert und Arbeitsabläufe hinsichtlich Beratung/Training und Umsetzung von FDM-Maßnahmen entwickelt. Diese sollen dazu dienen 
Klarheit, Akzeptanz und Effektivität zwischen allen Akteuren zu schaffen. Ziel ist, dass FDM Teil der Forschungspraxis wird.

Tobias Müllerleile (Marburg) sprach über „Forschungsdatenmanagement an Hochschulbibliotheken. Eine praxisbasierte Positionsbestimmung auf Basis der Erfahrungen aus dem HeFDI-Projekt" aus der Perspektive der Universitätsbibliothek Marburg. Hier wurden einerseits Diskussionen um die Initiative zum Aufbau einer Nationalen Forschungsdateninfrastruktur (NFDI) geführt, andererseits wurde die Rolle erörtert, die dabei die Bibliotheken einnehmen können, wobei auch von der Kooperation im Verbundprojekt „Hessische Forschungsdateninfrastrukturen“ (HeFDI), in dem eng und bedarfsorientiert mit der Forschung zusammengearbeitet wird, berichtet wurde. Fazit war, dass eine agile Arbeitsweise hilfreich ist und dass digitale Forschung ganzheitlich gesehen werden muss.

„FORSCHUNGSDATEN UND IHRE COMMUNITIES“ lautete der Titel von zwei weiteren Sessions.

Der Beitrag von Fiona Greig (Surrey) beschäftigte sich in ihrem Vortrag "Challenging assumptions and unlocking the last treasure trove of scholarship" mit dem zum Teil noch ungenutzten Potential an Abschlussarbeiten in Repositorien, die (noch) nicht öffentlich zugänglich sind. Auch derzeit noch nur analog vorhandene Arbeiten werden zunehmend digitalisiert und zur Verfügung gestellt.

Aline Frank (Bern) präsentierte ihren Vortrag unter dem Titel „Ein zweites Leben für Forschungsdaten: Wie Bibliotheken alte Forschungsdaten ins Rampenlicht bringen“. Der Fokus lag dabei auf Daten, die in früheren Forschungsprojekten erhoben worden waren, die aber nie öffentlich zugänglich gemacht wurden. Die Referentin führte in ihrer Abschlussarbeit im MAS-Studiengang Bibliotheks- und Informationswissenschaft eine Umfrage durch unter Forschenden der Naturwissenschaften an der Universität Bern und testete die nachträgliche Veröffentlichung von Daten, indem sie eigene Forschungsdaten zur Publikation vorbereitet und einreicht. Außerdem entwickelte sie Orientierungshilfen für Forschende.

Petra Buchholz (Berlin) sprach über „Forschungsdatenkompetenz erhöhen. Ein Train-the-Trainer-Programm zum Thema Forschungsdatenmanagement". Aufgrund der Knappheit der Personalressourcen und des gestiegenen Beratungs- und Schulungsbedarfs bzgl. FDM wurde das im Rahmen des Projekts FDMentor („Erarbeitung generalisierter Strategien und Lösungen für das Forschungsdatenmanagement unter Einbeziehung bestehender Expertise an universitären Zentraleinrichtungen") entwickelte Train-the-Trainer Programm verwendet, um weitere Personen in die Schulungsaktivitäten einzubeziehen. Das Programm ist interaktiv und vermittelt Grundlagen- 
kenntnisse in den wichtigsten Themenbereichen des Forschungsdatenmanagements. Eine Frage war, wie Bibliotheken hier offensiv werden und geeignete Services anbieten können.

In der zweiten Session zum Thema „FORSCHUNGSDATEN UND IHRE COMMUNITIES“ sprach Ingeborg Verheul (Amsterdam) unter dem Titel „Besser unterwegs mit Karotten und Süßigkeiten: Warum Zusammenarbeit im Forschungsdatenmanagement sich lohnt" über die seit 2015 existierende Koordinierungsstelle für FDM LCRDM (The National Coordination Point Research Data Management, https://www.Icrdm.nl/en). Sie soll auf politischer Ebene die Reproduzierbarkeit der Forschung und die bessere (Wieder-)Nutzung von Daten fördern und die Kooperation zwischen den Forschungsinstituten sicherstellen. Derzeit arbeiten bereits mehr als 100 ExpertInnen aus verschiedenen Forschungseinrichtungen (Universitäten, Universitätskliniken, Hochschulen, usw.) in den LCRDM zusammen, um unterschiedliche Forschungsdatenthemen auf eine nationale Ebene zu bringen. Die in den Niederlanden bereits etablierten Data Stewards sollen durch die Vernetzung von Bibliothek, IT und Politik den Forschenden damit bessere Unterstützung anbieten können. Seit 2017 gibt es in den Niederlanden auch den National Plan Open Science (NPOS), die unterschiedlichen Dachverbände sind hier gut aufeinander abgestimmtabgestimmt und die Forschungseinrichtungen arbeiten eng zusammen.

Gerald Jagusch (Darmstadt) beschäftigte sich mit dem Thema „Neues Archipel oder gemeinsame Verstetigung? Wie Landesinitiativen zu Forschungsdatenmanagement und NFDI verzahnt werden könnten - und sollten". Fazit ist, dass mehr Ressourcen nötig sind, um ein effektives FDM anbieten zu können - und auch mehr Awareness.

Beim Vortrag zum Thema „RISE-DE: Ein strukturierter, dialogischer Prozess zur Bewertung, Bedarfsermittlung und Strategieentwicklung für institutionelle Forschungsdatendienste" von Niklas Hartmann (Potsdam) stand das im Rahmen des BMBF-Verbundprojektes FDMentor angepasste RISE Framework des Digital Curation Centres (UK) im Mittelpunkt. RISE-DE kann für einen umfassenden, strukturierten und Stakeholder-orientierten dialogischen Prozess zur Selbstbewertung bestehender Dienste, zur Ermittlung der Bedarfe und zur Entwicklung einer Strategie eingesetzt werden. Es ist für den Einsatz an Hochschulen, die beim institutionellen Forschungsdatenmanagement noch ganz am Anfang stehen gedacht, aber auch für bereits weiter fortgeschrittene Einrichtungen.

In der Session „FORSCHUNGSDATEN IN DEN HUMANITIES“, moderiert von Andreas Brandtner (Berlin), wurde zunächst von Marina Lemaire (Trier) unter dem Titel „Koordiniert - interdisziplinär - über den Tellerrand hinaus. 
Die FuD-Philosophie für erfolgreiche nachhaltige Softwareentwicklung" von den Unternehmungen des Servicezentrums eSciences in Trier berichtet. Da Softwareanwendungen oft nur für einen bestimmten Forschungskontext passen und oft nach Projektende kein entsprechender Support mehr möglich ist, setzt man dort auf ein strategisches Vorgehen bei der Entwicklung und dem Regelbetrieb von Forschungssoftware, um einen nachhaltigen Betrieb solcher Infrastrukturen zu gewährleisten. 30 Projekte nutzen derzeit die virtuelle Forschungsumgebung für die Geistes- und Sozialwissenschaften.

Susanne Blumesberger (Wien) stellte unter dem Titel „Repositorien mehr als Datenspeicher? Möglichkeiten und Herausforderungen" das Repositorium Phaidra und das sich daraus entwickelte Datenmanagement vor.

Unter dem Titel „Forschungsdaten-Policies nach Maß“ stellte Bea Hiemenz (Berlin) das Forschungsdaten-Policy-Kit, ein modulares Baukastensystem für deutsche Universitäten und Hochschulen der Technischen Universität Berlin, vor. Forschungsdaten-Policies geben Universitäten und Hochschulen einen Orientierungsrahmen, der den Forschenden Transparenz und Klarheit verschafft, wie sie mit Forschungsdaten umgehen sollen. Gleichzeitig werden sie dabei unterstützt, die Anforderungen der Fördermittelgeber zu erfüllen. Forschungsdaten-Policies sind auch Management-Instrumente für die Einrichtung selbst. Im Rahmen des BMBF-Verbundvorhabens „FDMentor" hat die Technische Universität Berlin ein modulares Forschungsdaten-Policy-Kit mit Leitfragen und Textbausteinen entwickelt, das nachgenutzt werden kann. Zusätzlich gibt es einen Leitfaden, der die Universitäten und Hochschulen bei ihrem strategischen Vorgehen zur Implementierung einer Forschungsdaten-Policy an ihren Einrichtungen unterstützt.

Jürgen Rohrwild (Erlangen-Nürnberg) beschäftigte sich mit „eHumanities - interdisziplinär: ein Modellprojekt für Forschungsdatenmanagement in den digitalen Geisteswissenschaften" der Friedrich-Alexander-Universität Erlangen-Nürnberg. Ausgangspunkt des Vortrags war die Frage, wie (Meta-) Daten in den Geisteswissenschaften modelliert werden können, um projektspezifische Anforderungen zu erfüllen und fächerübergreifende Nachnutzung zu ermöglichen. Im Rahmen des vom Bayerischen Staatsministerium für Wissenschaft und Kunst geförderten Projektes „eHumanities - interdisziplinär" wurden konkrete (technische) Lösungen und Unterstützungsangebote erarbeitet. Dazu gehört die Weiterentwicklung bestehender Schnittstellen und Formate. Es wird vor allem auf die Bedürfnisse der digitalen Geisteswissenschaften eingegangen, angestrebt ist eine breite Nachnutzbarkeit der Lehrangebote als Open Educational Resources. Daten sollen möglichst keine Ecken und Kanten haben, für Daten aus den 
Digital Humanities (DH) ist das eher untypisch, was wiederum eine große Aufgabe für Bibliotheken darstellt.

Mit der Überführung von FDM-Projekten in den Dauerbetrieb am Praxisbeispiel RePlay-DH der Universitätsbibliothek Stuttgart setzte sich der Beitrag von Sibylle Hermann (Stuttgart) auseinander. Das Projekt RePlay$\mathrm{DH}$ hatte das Ziel eine Software zu entwickeln, die Forschende bei der Dokumentation ihrer Daten unterstützt. Forschungsprozesse werden im Hintergrund dokumentiert und zur Archivierung und Veröffentlichung vorbereitet. Eine Anbindung an verschiedene Repositorien wird vorbereitet, im Testbetrieb wird derzeit vom jeweiligen Use-Case ausgehend eine Beratung zur Annotation mit Metadaten angeboten.

„FORSCHUNGSDATENMANAGEMENT" war auch das Thema in einem von Janine Straka und Heike Neuroth (Potsdam) moderierten Hands-OnLab digital. Dabei wurde von Kerstin Wedlich-Zachodin (Karlsruhe) vorgestellt, wie „Aktives Datenmanagement mit RDMO“ betrieben werden kann. RDMO steht für die webbasierte Software Research Data Management Organiser (RDMO). Dabei handelt es sich um ein Tool, das die Erstellung von Datenmanagementplänen ermöglicht. Anhand eines strukturierten Fragebogens auf Deutsch und Englisch kann man die gesamte Lebensdauer der Daten beschreiben. Eine individuelle Anpassung an die eigenen Bedürfnisse ist möglich, auf GitHub können zusätzliche Elemente importiert werden.

Die Vorträge zeigen, dass die meisten wissenschaftlichen Bibliotheken mittlerweile Forschungsdatenmanagement betreiben. Aus zahlreichen Projekten haben sich bereits Services entwickelt, die je nach Kapazität der Institutionen umfangreichen Support für Forschende anbieten. Die Diskussionen haben aber auch gezeigt, dass in diesem innovativen Bereich aber auch noch viel zu tun ist.

Susanne Blumesberger

$$
* * * * * * *
$$

\section{NS-Provenienzforschung}

Das Panel „Provenienz und Restitution“ am 108. Deutschen Bibliothekartag erfreute sich auch dieses Jahr wieder eines regen Zulaufs. In sechs Vorträge wurden Ergebnisse, Herangehensweisen und aktuelle Herausforderungen thematisiert.

Maria Nüchter und Hans-Christian Pust präsentierten „Restitutionen von NS-Raubgut an der Württenbergischen Landesbibliothek Stuttgart" und damit die 
vorläufigen Ergebnisse, des dreijährigen Projekts, das noch bis Mai 2019 verlängert wurde. Mehr als 60.000 Bände wurden autopsiert. Mehrere herausragende Provenienzfunde wurden im Vortrag beschrieben, wie jener des 1933 nach Peru emigrierten Berliner Pathologen Max Hans Kuczysnki. Die Rückgabe erfolgte an den ehemaligen peruanischen Staatspräsidenten und Sohn, Pedro Pablo Kuczynski.

Elisabeth Geldmacher und Nadine Kulbe von der Sächsischen Landesbibliothek - Staats- und Universitätsbibliothek Dresden beschäftigten sich in ihrem Vortrag „Kein Ende in Sicht?! Voraussetzungen, Herausforderungen und Chancen der Suche nach NS-Raubgut in Erwerbungen ab 1945" mit den Erwerbungen nach Ende des Zweiten Weltkrieges. Dabei handelt es sich um Erwerbungen, die nicht automatisch in der NS-Provenienzforschung mitbedacht werden, wobei Raubgut-Bestände aufgrund von Enteignungen, Verlagerungen oder Ankäufe durch Antiquariate sehr wohl nach 1945 in Bibliotheken eingehen konnten. Die Kolleginnen führten als besondere Herausforderung hierbei die sogenannten „Parallelprovenienzen“ an. Sind mehrere Provenienzhinweise in einem Druckwerk vorhanden, wird die Klärung der Provenienzkette komplexer.

Der Vortrag von Regine Dehnel (Berlin) von der Staatsbibliothek zu Berlin - Preußischer Kulturbesitz beschäftigte sich mit „Auch die Sammelgebiete der Nationalbibliotheken in den Volksdemokratien werden beachtet. Die Zentralstelle für wissenschaftliche Altbestände". Die 1953 gegründete Stelle verteilte bis 1995 über acht Millionen Bücher aus Torsobibliotheken oder Dublettenbeständen in der DDR, aber auch zweitweise mit dem Ausland. Die Regularien zum Schriftentausch wurden erstmals 1976 festgelegt, zuvor wurden unterschiedliche Regelungen angewandt. Dehnel konnte in ihren Forschungen bisher nur wenige Fälle von Raubgut identifizieren.

Das Deutsche Zentrum Kulturgutverluste (DZK) wurde durch Sabrina Werner (Magdeburg) vertreten und stellte die neu entstehende Forschungsdatenbank vor. Das DZK wurde 2015 als Folgeinstitution der Arbeitsstelle Provenienzforschung begründet und stellt die staatliche Anlaufstelle für die NS-Provenienzforschung und zukünftig auch die Provenienzforschung an Kulturgütern in kolonialen Kontexten dar. In der bisherigen Forschungsdatenbank sind Projektergebnisse und -berichte aus DZK-geförderten Projekten nach Registrierung einsehbar. Das neue Webportal wird verstärkt Möglichkeiten zum Austausch und dem Ablegen von Quellenhinweisen bieten. Im Sinne der relationalen Datenbanken sollen alle Entitäten miteinander verbunden werden.

Die KollegInnen der Bayrische Staatsbibliothek Franziska Eschenbach und Stephan Kellner (München) präsentierten die virtuelle Ausstellung zur 
NS-Raubgutforschung, die in Kooperation mit "Google Arts and Culture" entstanden war. Zwar bestehen strenge Strukturvorgaben durch das Tool, dafür ermöglicht Google Arts \& Culture einen relativ einfachen OnlineAuftritt. Die virtuelle Ausstellung zur Provenienzforschung stellt die erste ihrer Art an Bibliotheken dar und ermöglicht den weltweiten Zugriff. Ein Highlight stellt das Interview mit dem Erben nach Alexander Dünkelsbühler dar, in dem ersichtlich wird, welche Bedeutung die Rückgabe eines Buches für ein Familienmitglied haben kann.

Als „internationalen Beitrag“, so die Moderatorinnen Jana Kocourek und Maria Kesting, trugen Olivia Kaiser und Markus Stumpf unter dem Titel „Bücher, Archivalien und museale Sammlungen. 15 Jahre Provenienzforschung in der Universitätsbibliothek Wien" die gewonnenen Erkenntnisse vor. Mehr als 400.000 Bücher wurden in der Hauptbibliothek und den Teilbibliotheken autopsiert, mehr als 60.000 für die weitere Recherche dokumentiert. In bisher 62 Fällen wurden Entscheidungen getroffen, davon erfolgten 25 Rückgaben an die rechtmäßigen Erblnnen oder RechtsnachfolgerInnen. Kaiser und Stumpf spannten dabei den Bogen von der Provenienzforschung in den Bibliotheken über das Archiv bis hin zu den Sammlungen der Universität Wien. Neben der Ausweisung der Provenienzfälle im bibliothekarischen Nachweisinstrument wurde auch die Vielfalt an Tätigkeiten (Forschung, Rückgaben organisieren, Tagungen, Publikationen, etc.) des Arbeitsbereiches aufgezeigt. Dabei versteht sich der Arbeitsbereich bei der Suche nach „fairen und gerechten“ Lösungen für die Beraubten und deren Erblnnen im Sinne der Washingtoner Prinzipien als wesentlichen Baustein der Erinnerungskultur an der Universität Wien. Aufgezeigt wurde, dass die Provenienzforschung mittlerweile weit über das Objekt selbst hinausführt und dennoch auch Spiegelbild bibliothekarischer Forschung und Aufgabengebiete ist.

Olivia Kaiser und Markus Stumpf

$* * * * * *$

\section{Hands-on Lab: Neue Geschäftsmodelle und Workflows im Open Access}

Ein neuerer Veranstaltungstyp, der Vortragenden die Möglichkeit bietet mit den Teilnehmenden in kleinen Gruppen intensiv an einem Thema zu arbeiten, sind so genannte „Hands-on Labs“. Diese lösten 2017 das bisherige Workshop-Format am Deutschen Bibliothekartag ab und sind in zwei Ausprägungen möglich: analog und digital (fokussiert auf die Umsetzung am Laptop/PC). Ein solches „Hands-on Lab analog“ wurde vom 
Nationalen Open-Access-Kontaktpunkt veranstaltet: Alexandra Jobmann (Bielefeld) stellte gemeinsam mit Dirk Pieper (Bielefeld) Ideen für „Neue Geschäftsmodelle und Workflows im Open Access"vor. Mittels Impulsvorträgen wurden drei mögliche Varianten vorgestellt, wie zukünftig aufOpen Access umgestellt oder Open Access allgemein gezahlt werden könnte. Drei Modelle wurden dabei erörtert:

1. Evidence Based Open-Access-Transformation: besonders für den geistes- und sozialwissenschaftlichen Bereich interessant, könnten über dieses Modell ebooks geflippt werden. Im Unterschied zu einer klassischen Evidence Based Acquisition wird das Werk am Ende nicht ge- sondern freigekauft.

2. Kooperatives Open-Access-Deutschlandkonsortium: angelehnt an das SCOAP3-Konsortium könnten hier gemeinsam mittels eines Abrechnungsmodells alle nationalen Zeitschriftenartikel eines OpenAccess-Verlags zentral bezahlt werden

3. Subscribe to Open: für die Open-Access-Transformation von Zeitschriften gedacht, sieht dieses Modell vor, dass die Abogebühren für den Freikauf eines vollständigen Jahrgangs genutzt werden. Jährlich kann wieder neu entschieden werden, ob der neue Zeitschriftenjahrgang Open Access oder subskriptionsbasiert zugänglich ist.

Die Modelle wurden in Kleingruppen diskutiert und jeweils einer SWOTAnalyse (Stärken/Schwächen/Risiken/Chancen) unterzogen. Die Diskussion profitierte besonders stark von der heterogenen Zusammensetzung des Publikums: Bibliothekarlnnen, Vertreterlnnen von Verlagen und Dienstleisterlnnen beteiligten sich aktiv in den Gruppen. Positiv wahrgenommen wurde von den Teilnehmenden auch, dass OA-Geschäftsmodelle von den Bibliotheken nicht nur passiv angenommen, sondern durch solche Veranstaltungen auch aktiv mitgestaltet werden können.

Abgerundet wurde das Hands-on Lab mit einer kurzen Umfrage unter den Teilnehmenden. Dabei wurde festgelegt, welche weiteren Kriterien erfüllt sein müssen, um sich an einem der Geschäftsmodelle zu beteiligen. Besondere Erwähnung fanden hier: eine nachhaltige Open-Access-Transformation, Kostentransparenz und Planbarkeit, Verteilungsgerechtigkeit zwischen den Institutionen, funktionierende AutorInnenidentifikation, vorhandene Qualitätssicherung und dass das Modell im Einklang mit bestehenden Policies der Institutionen oder Förderer steht. 


\section{Hands-on Lab digital: Einsatz von Linux-Tools unter Windows für Bib- liothekarInnen}

Ein Hands-on Lab ganz anderer Art war der „Einsatz von Linux-Tools unter Windows für Bibliothekarlnnen", abgehalten von Uwe Dierolf (Karlsruhe). Ziel des Trainings - welches an der KIT-Bibliothek bereits 2017 für Mitarbeiterlnnen durchgeführt wurde - war die Heranführung von Bibliothekarlnnen an einfache Datenverarbeitungsmöglichkeiten mithilfe von Linux-Tools, die auch unter Windows eingesetzt werden können. Dazu muss Linux nicht in einer VirtualBox laufen, für die meisten Anwendungsfälle reicht der Einsatz der Bash. Die Bash nimmt Befehle direkt auf der Kommandozeile entgegen und sie erfreut sich besonders bei fortgeschrittenen AnwenderInnen großer Beliebtheit. Im Hands-on Lab wurde die Software MobaXterm (https://mobaxterm.mobatek.net/ download-home-edition.htm) eingesetzt und dafür wurden den Teilnehmerlnnen zunächst einfache Kommandozeilen-Befehle (pwd, dir, cd) für die Bewegung in den Verzeichnissen und im Umgang mit Dateien (cat, less, grep, sort etc.) erklärt. Mit diesem Basiswissen wurden mehrere Beispiele gemeinsam an den eigenen Laptops durchgegangen: exportierte Mailadressen wurden ausgeschnitten und gelistet, MAB-Datensätze nach Jahren gruppiert, Daten von Besucherzählern ausgewertet und XML und JSON-Daten wurden reduziert und gezählt. Diese Veranstaltung zeigte nicht nur, wie effizient bereits mit einigen Grundkenntnissen gearbeitet werden kann, sondern auch, dass eine gewisse Automatisierung von Abläufen mit wenig Aufwand möglich ist. Offen blieb allein die Frage der Anwendungsgebiete - die Beantwortung obliegt wohl der Kreativität der Teilnehmenden.

Magdalena Andrae

$* * * * * *$

\section{Hands-on Lab digital: DINI Metadata Crunch}

Einen ähnlichen Zugang hatte ein von Najko Jahn (Göttingen) moderiertes „Hands-on Lab digital“, das sich die Vermittlung von IT-Kenntnissen zum Ziel gesetzt hatte. „DINI Metadata Crunch: Praktische Schritte zur Nachnutzung von Metadaten aus DINI-zertifizierten Repositorien" zeigte drei (bekannte und 
weniger bekannte) Tools, die bei der Datenverarbeitung von Metadaten aus Repositorien helfen können. Organisiert wurde das Hands-on Lab von der DINI AG Elektronisches Publizieren, einführend wurde daher auch auf den Entwurf für das DINI-Zertifikat für Open-Access-Publikationsdienste 2019 eingegangen, welche zu dem Zeitpunkt noch zur Diskussion stand (https://doi.org/10.5281/zenodo.2575346).

Danach wurden Catmandu, OpenRefine und R kurz präsentiert, im Anschluss teilten sich die Anwesenden in drei Gruppen auf um eines der Tools näher kennenzulernen: Michaela Voigt (OpenRefine), Vitali Peil (Catmandu) und Najko Jahn (R) zeigten konkrete Anwendungsbeispiele her und berichteten von den Möglichkeiten, Vorteilen, sowie auch Grenzen. Im weiteren Verlauf wurde dieses so aufgebaute Wissen wieder von einer Teilnehmerin/ einem Teilnehmer in die große Gruppe zurückgetragen. In der kurzen Zeit von 2,5h konnten die jeweiligen Themen leider nur angerissen werden, es wurde jedoch ein guter Überblick über die Tools und ihre unterschiedlichen Anwendungsfälle geboten.

Überraschenderweise war der Andrang bei diesem Hands-on Lab eher gering, wovon man in den Gruppenarbeiten wiederum profitieren konnte. Dieses Hands-on Lab kann von Interessierten auch auf Github nachgelesen werden, dort finden sich die Anwendungsbeispiele zum Nachmachen: https://github.com/njahn82/dini_md_crunch

Magdalena Andrae

$* * * * * *$

\section{Qualitätsmanagement-Forum 2019}

Am 18. März 2019 fand in Leipzig eine öffentliche Arbeitssitzung der Managementkommission von dbv und VDB, moderiert von Cornelia Vonhof (Stuttgart), zum Thema Qualitätsmanagement statt. Zielgruppe der Veranstaltung, die bereits im Rahmen der Bibliothekartage in Frankfurt am Main 2017 und in Berlin 2018 erfolgreich durchgeführt worden war, waren Vertreterinnen und Vertreter von Einrichtungen, an denen bereits Qualitätsmanagement betrieben wird. Das „Qualitätsmanagement-Forum 2019“ bot zunächst den ca. 25 Teilnehmerinnen und Teilnehmern Gelegenheit, über ihre Erfahrungen bei der Implementierung und der Weiterentwicklung des an ihren Einrichtungen eingesetzten Qualitätsmanagementsystems zu berichten. Bei aller Unterschiedlichkeit der angewendeten Systeme zeigte sich in einigen Bereichen eine sehr ähnliche Problemlage. Bei der Imple- 
mentierung eines Qualitätsmanagementsystems sind vor allem Prozessdokumentation und betriebswirtschaftliches Denken wichtige Herausforderungen. Wurde ein System einmal gut zum Laufen gebracht, ist Durchhaltevermögen gefragt, um den Qualitätsmanagementgedanken lebendig zu halten. Zur Motivationsförderung beitragen könnten Bemühungen, das Potenzial von Qualitätsmanagement auszuschöpfen, aber auch die Einführung eines Anreizsystems.

Einen guten Einblick in die konkreten Herausforderungen, denen sich Einrichtungen zu stellen haben, die eine Zertifizierung anstreben, vermittelten zwei kurze Präsentationen. Während die Stadtbücherei Geislingen an der Steige das Ziel „Ausgezeichnete Bibliothek“ als eine der ersten drei Bibliotheken deutschlandweit bereits 2011 erreicht hat und sich nunmehr im Dreijahreszyklus einer Rezertifizierung stellen muss, wurde im Erfahrungsbericht einer Fachhochschulbibliothek deutlich, wie aufwändig und langwierig sich die Vorbereitungsphase eines Zertifizierungsverfahrens gestalten kann.

Anschließend wurde am Beispiel der Universitätsbibliothek Bern dargestellt, wie qualitätssteigernde Maßnahmen auch außerhalb eines normierten Zertifizierungsverfahrens erfolgreich umgesetzt werden können. In Bern wurde eine Arbeitsgruppe eingesetzt, die Qualitätsmanagementstandards für die Kundenservices entwickeln sollte. Nach einer Umfrage unter den Mitarbeiterinnen und Mitarbeitern wurden drei Workshops durchgeführt, um die Themenbereiche, bei denen Verbesserungen angestrebt werden, zu identifizieren. Entwickelt wurden zwölf Qualitätsstandards, die auf Kärtchen veranschaulicht wurden. Unter Nutzung dieses in der Praxis sehr gut einsetzbaren Hilfsmittels wurden die festgelegten Standards an der Hauptbibliothek und den 33 Teilbibliotheken der Universitätsbibliothek Bern implementiert.

Für das „Qualitätsmanagement-Forum 2019“ ist festzuhalten, dass die vorgestellten unterschiedlichen Ansätze von Qualitätsmanagement, aber auch der intensive Erfahrungsaustausch über die Praxis an den einzelnen Institutionen wiederum wertvolle Anregungen für die Teilnehmerinnen und Teilnehmer mit sich gebracht haben. Das Qualitätsmanagement-Anwendertreffen hat sich somit für QM-Zuständige an öffentlichen und wissenschaftlichen Bibliotheken als attraktiver Fixpunkt im Programm der Deutschen Bibliothekartage etabliert. 


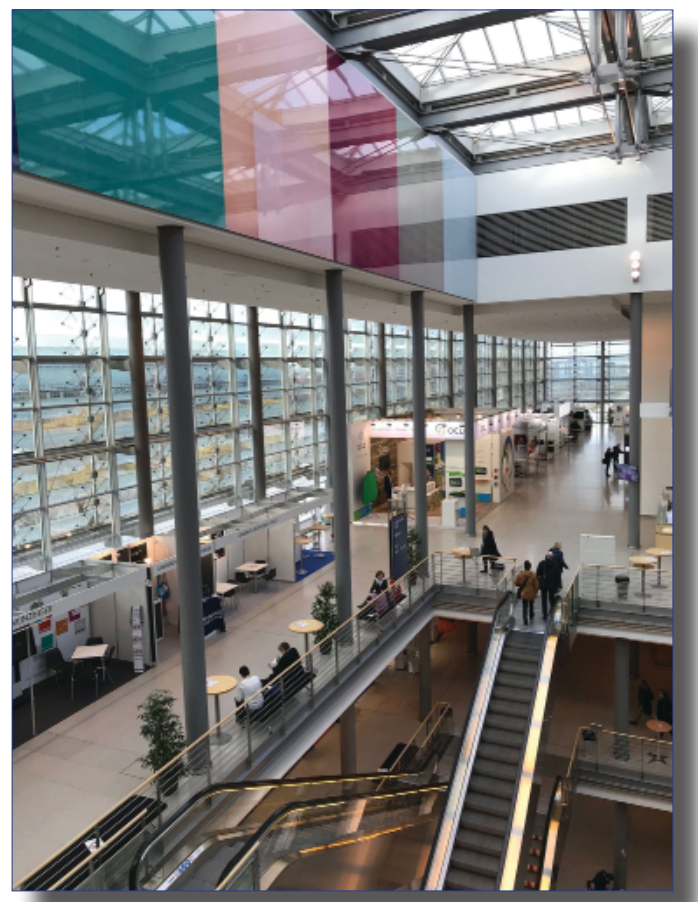

Abb. 3: Congress Center Leipzig: Blick ins Foyer (Foto: Susanne Blumesberger 2019)

\section{Barrierefreiheit in (digitalen) Bibliotheken}

Die auf dem Deutschen Bibliothekartag 2018 gegründete Arbeitsgruppe AG Barrierefreiheit in (Digitalen) Bibliotheken ist ein offenes Forum für den Austausch von Informationen und konkreten Erfahrungen bei der Umsetzung von Barrierefreiheit in Bibliotheken. Themen sind unter anderem rechtliche Grundlagen, Standards, Formate, barrierefreier Zugang zu Informationen und Informationstechnologien, barrierefreie Gestaltung von Veranstaltungen und Bibliotheksgebäuden. Dazu gehört aber auch die Inklusion von Menschen mit Behinderungen als Mitarbeitende in der Bibliothek.

Thematisiert wurden in der von Christiane Felsmann (Leipzig) und Anne Sieberns (Berlin) moderierten öffentlichen Arbeitssitzung unter anderen der Marrakesch-Vertrag, der lesebehinderten Personen den Zugang zu Informationen erleichtern soll. Dazu zählen nicht nur blinde und sehbehinderte, sondern auch kognitiv oder motorisch beeinträchtigte Personen. Bibliotheken sind jene Stellen, die diesen Zugang gewähren können. 
Die Universitätsbibliothek Leipzig ist Vorreiterin beim Thema Barrierearmut. Barrierefreiheit dient nicht nur Menschen mit Beeinträchtigungen, sondern allen. Eine einfachere Sprache, gute Strukturierungen, Bildbeschreibungen, scharfe Kontraste usw. sind dabei nötig. Ein sauberer Code ist ebenfalls nötig. Eine Opensource-Software soll dabei unterstützen, allerdings wird für die Weiterentwicklung eine hohe Anzahl an BenutzerInnen benötigt. Vor Kurzem wurde der European Accessibility Act unterzeichnet.

Den Usern wird ein Webformular für barrierefreie Literatur angeboten. Vorgestellt wurde auch der sehgeschädigtengerechte Katalog „Online Sehkon" (https://www.ub.uni-dortmund.de/sehkon/), der an derTechnischen Universität Dortmund betreiben wird und der Medien für Sehgeschädigte mit zitierfähig umgesetzter Literatur im deutschsprachigen Raum verzeichnet.

Derzeit sind über 400 Nutzerlnnen angemeldet. Ein internationaler Austausch wird angestrebt. Allerdings werden Personen mit Dyslexie noch nicht berücksichtigt.

Allgemein lässt sich sagen, dass trotz zahlreicher Vorgaben, Barrierefreiheit in den wenigsten Bibliotheken umfassend umgesetzt wird.

Susanne Blumesberger

$* * * * * *$

\section{Podiumsdiskussion „Personal \& Kompetenzen - Wie gut sind wir?“}

Martin Elsner (Bundesinstitut für Berufsbildung), Matthijs van Otegem (Erasmus University Rotterdam), Ton van Vlimmeren (Öffentliche Bibliothek Utrecht), Charlotte Bauer (Universitätsbibliothek Leipzig) und Volker Heller (Zentral- und Landesbibliothek Berlin) stellten sich in einer Podiumsdiskussion zum Thema „Personal \& Kompetenzen - Wie gut sind wir?" der Frage welche Strategien man benötigt, um dem Fachkräftemangel an Bibliotheken wirksam begegnen zu können. Interessant war hier der unterschiedliche Zugang zum Thema. Otegem berichtete beispielsweise, dass er bei Bewerbungsgesprächen nicht mehr dabei sei, die Mitarbeiterlnnen selbst suchen neue Kolleginnen und Kollegen aus. In den Niederlanden gibt es auch keine Bibliotheksschulen mehr. Einig war man sich, dass Bibliotheken in Zukunft kreative Räume benötigen und dass neue Kompetenzen gebraucht werden, vor allem im kommunikativen Bereich. Es gibt derzeit noch kein zukünftiges Berufsbild von Bibliothekarinnen und Bibliothekare, wichtig ist jedoch auch Quereinsteigerinnen und Quereinsteiger zu berücksichtigen und flexibel zu bleiben 
und Praxis und Ausbildung weiter zu verschränken. Es ist in der heutigen Zeit unmöglich, „fertige“ Bibliothekarinnen auszubilden.

Susanne Blumesberger

$* * * * * *$

\section{Podiumsdiskussion „Herausforderungen bewältigt?“}

In der von Andreas Mittrowann (Strategieberater aus Recklinghausen) moderierten Podiumsdiskussion „Herausforderungen bewältigt?" diskutierten Petra Köpping (Sächsische Staatsministerin für Gleichstellung und Integration), Dietrich Nelle (bis 2018 interimistischer Leiter der Zentralbibliothek für Medizin), Hella Schwemer-Martienßen (Direktorin der Hamburger Bücherhallen) und Aat Vos (Architekt und Creative Guide aus den Niederlanden), über Herausforderungen für Bibliotheken. Thematisiert wurde unter anderem inwieweit Bibliotheken als unpolitische Räume, wo diskutiert werden kann, gesehen werden. Vos knüpfte an den Appell von Habermas an, freie Räume zu schaffen um Demokratie wachsen zulassen. Man braucht öffentliche Räume, die aber auch Sicherheit geben. Wichtig ist auf jeden Fall in der Zukunft aktiver auf Bibliotheksnutzer einzugehen, es wird Mut zur Veränderung verlangt, Veränderungen beschleunigen sich immer mehr und es wird immer schwieriger werden auf diese zu reagieren. Köpping rief dazu auf, die Erfahrungen der Ostdeutschen und Personen anderer osteuropäischer Länder in Diskussionen einfließen zu lassen, denn sie haben Veränderungen am eigenen Leib gespürt. Einig war man sich auch darüber, dass vorhandene Kompetenzen mehr wertgeschätzt werden sollten. Laut Vos sind dabei Veränderungen der Kompetenzen nötig, beispielsweise arbeiten in Norwegen sechs Personen an einer Jugendbibliothek, die keine ausgebildeten Bibliothekare sind. Eine gute Personalentwicklung wird hier immer wichtiger, die Ausbildung sollte weniger verschult sein, gleichzeitig sollten Bibliothekarlnnen besser bezahlt werden. Ausschlaggebend ist auch eine gut funktionierende Zusammenarbeit, die nur wirklich funktionieren kann, wenn auch Strukturen verändert werden. Vos zeigte anhand der Maslowschen Bedürfnishierarchie, dass laut einer Umfrage auch bei den Bibliotheksnutzerinnen und Benutzern die Ansprüche immer weiter steigen.

Susanne Blumesberger 
Mag. Sebastian Aigner, MSc Die Österreichische Bibliothekenverbund und Service GmbH (OBVSG) E-Mail: sebastian.aigner@obvsg.at

MMag. ${ }^{a}$ Magdalena Andrae ORCID: https://orcid.org/0000-0001-9174-7449 Technische Universität Wien, Universitätsbibliothek E-Mail: magdalena.andrae@tuwien.ac.at

Mag. Bruno Bauer ORCID: https://orcid.org/0000-0002-4729-331X Medizinische Universität Wien, Universitätsbibliothek E-Mail: bruno.bauer@meduniwien.ac.at

Dr. ${ }^{\text {in }}$ Susanne Blumesberger ORCID: https://orcid.org/0000-0001-9018-623X Universität Wien, Bibliotheks- und Archivwesen E-Mail: susanne.blumesberger@univie.ac.at

Mag. ${ }^{a}$ Olivia Kaiser ORCID: https://orcid.org/0000-0002-1264-4853 Universität Wien, Bibliotheks- und Archivwesen E-Mail: olivia.kaiser@univie.ac.at

Mag. Markus Stumpf, MSc ORCID: https://orcid.org/0000-0003-4946-9988 Universität Wien, Bibliotheks- und Archivwesen E-Mail: markus.stumpf@univie.ac.at 